\title{
Gastrointestinal stromal tumor (GIST) recurrence following surgery: review of the clinical utility of imatinib treatment
}

This article was published in the following Dove Press journal:

Therapeutics and Clinical Risk Management

21 September 2010

Number of times this article has been viewed

\author{
Isabelle Deshaies' \\ Jovenel Cherenfant ${ }^{1}$ \\ Niraj J Gusani' \\ Yixing Jiang ${ }^{2}$ \\ Harold A Harvey ${ }^{2}$ \\ Eric T Kimchi' \\ Jussuf T Kaifi' \\ Kevin F Staveley-O'Carroll' \\ 'Program for Liver, Pancreas and \\ Foregut Tumors, Department of \\ Surgery, Penn State College of \\ Medicine, Hershey, Pennsylvania, USA; \\ ${ }^{2}$ Department of Medical Oncology, \\ Penn State College of Medicine, \\ Hershey, Pennsylvania, USA
}

\begin{abstract}
Gastrointestinal stromal tumor (GIST) is the most common mesenchymal tumor of the gastrointestinal tract. Surgery with complete removal of the tumor is the primary treatment for resectable GIST and the only chance of cure. However, recurrence after surgery is common. The 2 main prognostic factors are the mitotic activity and the size of the tumor. Tumor rupture is also a risk factor for postoperative recurrence, and extra care should be taken while manipulating this soft and friable tumor. Imatinib mesylate (IM, Gleevec ${ }^{\circledR}$, Novartis, Basel, Switzerland) is a tyrosine kinase inhibitor and was first studied in the palliative setting for metastatic GIST patients in the year 2000. It is now the cornerstone of metastatic GIST treatment. IM also plays an important role as an adjuvant treatment for resectable GIST and has been shown to increase the recurrence-free survival in phase III studies. However, some points remain to be clarified. Notably, the ideal duration of adjuvant IM after surgery is still unclear. It is also difficult to determine the exact place of surgery in metastatic or recurrent GIST patients in the IM era. A multidisciplinary approach is, therefore, mandatory to offer GIST patients the best treatment available.
\end{abstract}

Keywords: Gleevec, adjuvant, neoadjuvant, metastasis, sunitinib

\section{Introduction}

Gastrointestinal stromal tumor (GIST) is the most common mesenchymal tumor of the gastrointestinal tract. For years, the only effective treatment available was surgery. Although surgery has a high rate of cure for low-risk GIST, it is seldom curative for high-risk tumors since the postoperative recurrence rate for GIST with high-risk features is usually high. In 1998, Hirota et al demonstrated the central role of a gainof-function mutation of the kit gene in this disease process. ${ }^{1}$ This proto-oncogene encodes a transmembrane receptor, CD117. This discovery has led to a revolution in the treatment of this disease: the introduction of imatinib mesylate (IM, Gleevec ${ }^{\circledR}$, Novartis, Basel, Switzerland). This molecular-targeted agent, initially developed to treat chronic myeloid leukemia, is an inhibitor of multiple receptor tyrosine kinases, including the receptor CD117. In the year 2000, IM was first used as a salvage therapy in a patient with metastatic GIST who had failed multiple lines of treatment. An impressive response was observed with reduction in tumor volume and normalization of 18F-fludeoxyglucose (FDG) activity on positron emission tomography (PET). ${ }^{2}$ This success led to the initiation of clinical trials to further evaluate the efficacy of IM in GIST treatment.

IM is now a cornerstone in the treatment of GISTs. In this review, we provide a summary of the initial experience in GIST management in the pre-IM era. The mechanism 
of action, pharmacology, safety profile, tolerability, and efficacy in clinical studies of IM are also discussed. Finally, based on the current data, the role of surgery in conjunction with molecular treatment is investigated.

\section{GIST epidemiology and pathophysiology}

GISTs arise from the interstitial cells of Cajal. The literature before the year 2000 on GIST incidence is unreliable because this tumor was often misclassified as a smooth muscle tumor or other mesenchymal tumor. ${ }^{3}$ In a population-based study conducted in western Sweden, with in-depth pathological and histological reviews, the annual incidence of GIST was found to be 14.5 cases per million. By extrapolating this number, we can estimate that about 3,000-5,000 new cases of GIST are probably diagnosed each year in the United States. ${ }^{4}$

GIST occurs mostly in the stomach $(60 \%-65 \%)$ or small intestine $(25 \%-35 \%)$ but can arise anywhere along the gastrointestinal tract..$^{4-7}$ It is mostly a rare sporadic tumor seen in older adults (median age 55-60 years). Familial GIST is rare but can be seen in patients with neurofibromatosis type I (multiple small intestinal tumors) or Carney's triad (gastric epithelioid GIST, pulmonary chondroma, and extra-adrenal paraganglioma). Histologically, 95\% of GISTs express the transmembrane receptor CD117, which is the main immunohistological marker. ${ }^{5}$ In tumors lacking a kit mutation, approximately $35 \%$ have a gain-of-function mutation of the platelet-derived growth factor receptor alpha, a related tyrosine kinase receptor. ${ }^{8}$ This mutation is nearly exclusive for gastric CD117-negative GISTs.

The clinical behavior of GISTs can vary from a benign to a highly aggressive course. The 2 main prognostic factors are the mitotic activity and the size of the tumor. More than 5 mitoses per 50 high-power fields (HPF) and size greater than $10 \mathrm{~cm}$ are almost uniformly identified as factors associated with poor outcomes after surgery. ${ }^{4,6,9,10}$ Other factors such as nongastric primary tumor location, exon 9 mutation, or "wild type" kit also appear to be deleterious prognostic factors. ${ }^{9,11-13}$

\section{General management}

For primary GISTs, only complete surgical resection offers a possibility of cure. The initial patient evaluation should include a computed tomography (CT) scan of the abdomen and pelvis and chest imaging. A PET scan and an endoscopic ultrasound can sometimes be included. A biopsy is not mandatory before surgery, except if neoadjuvant treatment is considered or if the differential diagnosis includes lymphoma. If a biopsy is required, the endoscopic route is preferred to decrease the risk of tumor seeding. The main goal of surgery is to remove the tumor with negative margins. A 2-cm margin should be sought. A regional lymphadenectomy is generally not required since GIST rarely $(<5 \%)$ metastasizes to lymph nodes. ${ }^{7,14,15}$ GIST rarely invades other organs and can usually be lifted away from surrounding structures. When this is not the case, involved organs should be resected en bloc with the specimen. Extra care should be taken while manipulating the tumor because GISTs are soft and fragile in nature, and intraperitoneal rupture or bleeding is associated with high risk of postoperative recurrence. ${ }^{9,16}$

IM is the cornerstone of metastatic GIST treatment. Recurrent GIST should be considered as an equivalent of metastatic tumor. The usual starting dose of IM is $400 \mathrm{mg}$ daily. ${ }^{16}$ This treatment is associated with a good disease control rate, but complete response rate is low. As demonstrated in 2 phase III studies, the complete response rate was 5\%, while the partial response rate reached between $40 \%$ and $45 \%$. Approximately, $25 \%-32 \%$ of patients experienced stable disease. ${ }^{17,18}$ If no major toxicity occurs, IM should be continued until disease progression. In the setting of disease progression, a dose escalation should be considered. When the tumor develops resistance to IM, sunitinib malate (SM, Sutent $^{\circledR}$, Pfizer, New York, NY, USA) is an effective second-line therapy. ${ }^{19}$ The integration of surgery in metastatic disease needs to be carefully outlined in the context of medical therapy. Nonetheless, data to guide surgical decision making, such as timing for intervention, are very limited.

\section{Surgery in the pre-IM era}

Historically, surgery was the only effective treatment available for GISTs. However, the risk of postoperative recurrence was fairly high in tumors with high-risk features. Pierie et al demonstrated a 5 -year overall survival (OS) of $42 \%$ in GIST patients with complete gross resection compared with $9 \%$ if the excision was incomplete. ${ }^{14}$ DeMatteo confirmed a similar 5-year disease-specific survival of $35 \%$ in 200 patients who had surgical resection for primary or metastatic GIST. ${ }^{7}$ In the population-based Swedish study presented earlier, recurrences were in 3 out of 169 patients with low- or intermediate-risk GIST and in 35 out of 53 patients with high- or very high-risk GIST. ${ }^{10}$

The above studies dealt with the outcomes after surgery for primary or recurrent GIST. Rutkowski et al addressed the question of recurrence after primary tumor resection only, demonstrating a 5-year disease-free survival (DFS) of $37.8 \%$ in patients with resected primary GISTs. As shown in the multivariate analysis, more than 5 mitoses per $50 \mathrm{HPF}$, 
tumor size greater than $5 \mathrm{~cm}$, nongastric primary location, male sex, and R1 resection or tumor rupture were associated with adverse outcomes. Patients with low-risk GIST reached a 5-year DFS of $96 \%$, whereas those with intermediate- or high-risk tumors frequently had much lower DFS, 54\% and $20 \%$, respectively. ${ }^{9}$ A study on resected metastatic-only GIST patients demonstrated a median survival of 19 months and a 5 -year OS of $25 \% .^{20}$

The pre-IM era literature clearly demonstrates an elevated risk of recurrence in patients presenting with intermediate- or high-risk tumors and metastatic disease treated by surgery only. It is clear that surgery alone is not sufficient to provide long-term survival for a vast number of GIST patients.

\section{The IM era}

The first studies on IM were conducted for metastatic GIST patients in the beginning of the year 2000. The initial phase II studies demonstrated a good tolerability and an encouraging response. Although complete response was low, partial response rate of approximately 54\%-67\% was reached. In addition, $18 \%-28 \%$ of the patients had stable disease. ${ }^{21-23}$ Thus, it can be concluded that more than $80 \%$ of patients on IM therapy would have clinical benefit.

The efficacy of higher dose of IM was assessed in 2 phase III studies. In the North American Sarcoma Intergroup S0033 trial, 746 patients were treated with either $400 \mathrm{mg}$ daily dose (standard dose) or $800 \mathrm{mg}$ daily dose of IM. No significant difference in progression-free survival was detected between the two doses used (18 months in $400 \mathrm{mg}$ and 20 months in $800 \mathrm{mg}$ group). Median OS was similar in both groups, with 55 months in the standard dose arm and 51 months in the higher dose arm. ${ }^{18}$ In the European Organisation for Research and Treatment of Cancer (EORTC) and Australian trial, a favorable DFS was documented in patients treated with the $800 \mathrm{mg}$ daily dose of IM. However, treatment interruption and dose reduction were more frequent. ${ }^{17}$ Subgroup analysis demonstrated the feasibility, safety, and efficacy of dose escalation upon disease progression. ${ }^{24}$ Interestingly, one subgroup analysis for each trial has also demonstrated that patients harboring exon 9 mutations frequently do not respond to standard dose of IM. A higher dose is usually required to control the disease. ${ }^{12,13}$

The impact of IM in the adjuvant setting was evaluated in patients with surgically resected primary GIST. The first phase II trial, ASCOSOG Z9000, was conducted in patients operated on for a primary GIST with high risk of recurrence (size $\geq 10 \mathrm{~cm}$, ruptured, intraperitoneal hemorrhage, multifocal GIST). The study demonstrated improvement of DFS and
OS when compared with historical controls. ${ }^{25}$ The subsequent phase III trial, ACOSOG Z9001, randomized a total of 708 patients to either 1-year therapy of $400 \mathrm{mg}$ daily of IM or placebo with a primary end point of OS and DFS. This trial was stopped prematurely because the interim analysis showed significant prolongation of DFS. At a median follow-up of 19.7 months, only $8 \%$ of IM-treated patients had recurrence compared with $20 \%$ of placebo-treated patients. The estimated 1-year DFS was $98 \%$ in the IM group compared with $83 \%$ in the placebo group. ${ }^{26}$ However, the OS was similar in both the groups, suggesting that recurrent GIST could be successfully rescued using IM. There are two other ongoing phase III trials. The EORTC trial 62024 randomizes patients with intermediate- or high-risk GIST to 2 years of adjuvant IM versus placebo. The Scandinavian Sarcoma Group XVIII trial analyzes the effect of IM adjuvant therapy for either 1 or 3 years. The study completed its accrual, and the results are awaited. These data will provide new insights into the duration of adjuvant therapy.

Preoperative (neoadjuvant) therapy with IM is an attractive approach especially if a diminution of the tumor size would allow a less extensive and less morbid surgery or if the tumor is not resectable. However, data from prospective studies are very scant. The results of the phase II RTOG 0132/ACRIN 6665 were recently published, demonstrating the feasibility of this approach. ${ }^{27}$ Fifty-two assessable patients, with advanced primary and metastatic/recurrent tumors, were treated with $600 \mathrm{mg}$ daily dose of neoadjuvant IM. Patients with responding tumors or stable disease were recommended surgical resection. Postoperatively, patients were further treated with adjuvant IM for 2 years. The median length of treatment before surgery was 65 days. The vast majority of patients had stable disease prior to surgery. Only $7 \%$ of patients with advanced primary tumor and $4.5 \%$ of patients with recurrent/metastatic GIST had tumor shrinkage. Postoperative complications did not appear to be worsened. Two-year progression-free survival was $83 \%$ for patients with advanced primary GIST and $77 \%$ for metastatic/recurrent GIST. In another retrospective study, 15 patients with unresectable or locally advanced primary GIST were treated with neoadjuvant IM for a median of 9 months. All patients experienced tumor size reduction, with a median shrinkage of $34 \%$. Three unresectable patients became resectable, and 7 patients had a less extensive surgery than initially planned. ${ }^{28}$

There is growing evidence that tumor response to IM is better assessed by metabolic imaging such as density modification on CT scan or Standardized Uptake Values $\left(\mathrm{SUV}_{\max }\right.$ ) on ${ }^{18}$ FDG-PET scan. ${ }^{29,30}$ Patients may present with stable disease 
according to standard Response Evaluation Criteria in Solid Tumors (RECIST) criteria but have a demonstrable response according to metabolic imaging. In the Radiation Therapy Oncology Group 0132 and American College of Radiology Imaging Network (RTOG 0132/ACRIN ) 6665 study, less than $8 \%$ of patients had tumor shrinkage. If tumor size reduction is sought, such as in patients presenting with initially unresectable tumors, there is some evidence that a longer treatment period is needed. However, for marginally resectable patients, the risk of progression under lengthy IM therapy must be considered. Larger studies with proper controls are needed to assess the usefulness of neoadjuvant treatment, especially in patients with a primary tumor that is initially resectable.

\section{Mode of action, drug resistance, and safety profile of IM}

GISTs carry a mutation in the kit gene located on chromosome 4. The gene encodes a transmembrane protein that serves as a receptor for the growth factor stem cell factor (SCF). The transmembrane protein has an intracellular domain containing the tyrosine kinase enzyme, which, once activated, propagates a cascade of activities ultimately causing cell division. The mutation in the kit gene causes uncontrolled activation of the tyrosine kinase independently of the growth factor SCF. The activation of the tyrosine kinase involves the binding of an adenosine triphosphate molecule to the active site of the tyrosine kinase. IM is one of the first generations of tyrosine kinase inhibitors that works by occupying the active site thereby preventing the phosphorylation and activation of the enzyme. ${ }^{31}$

IM is a 2-phenylaminopyrimidine derivative. Administered only orally, it has an oral availability of $98 \%$ in patients with normal hepatic function. Its absorption is not modified by food. It binds primarily to the albumin in plasma. Peak plasma levels occur 2-4 hours after oral administration. It is metabolized mainly by the cytochrome P450 system in the gut wall and liver. Its major metabolite is the N-demethylated piperazine derivate, which is also active. The volume of distribution of IM is not well studied. The elimination half-life for IM and its active metabolite averages 18 and 40 hours, respectively. IM is mainly excreted by fecal elimination via the bile, mostly as metabolites. A quarter of the dose is excreted unchanged. Age does not seem to have any significant effect on pharmacokinetics. The dosage needs to be adjusted in severe hepatic or renal impairment. ${ }^{16,32,33}$

In a group of phase I, II, and III studies, ${ }^{18,23,34}$ the most common toxic effects of IM at $400 \mathrm{mg}$ once daily included nausea, vomiting, edema, skin rash, and bone marrow toxicity. These side effects were reported to be tolerable. A small percentage of patients with large bulky tumors experienced significant tumor hemorrhage. ${ }^{21}$ Hepatotoxicity has also been observed in about $2 \%-5 \%$ of patients. The toxicity is usually mild and resolves with dosage reduction or discontinuation of IM. ${ }^{21,35}$ Table 1 provides a summary of the management of IM toxicity and side effects.

Although tolerable, the impact of these symptoms on the quality of life of GIST patients treated with IM has not been well studied. The Van Glabbeke group reported extensive data on predicting toxicities on 942 GIST patients treated with IM using the Common Toxicity Criteria (CTC) grading scale. ${ }^{36}$ However, caution should be taken in extrapolating these data to patients' quality of life because they are largely based on pathophysiological parameters, and abnormal pathophysiological values do not always translate into poor quality of life. There remains a need for a well-designed study that analyzes the impact of IM on patients' quality of life, encompassing patients' satisfaction, acceptability, and adherence.

Although IM is effective against GIST, acquired drug resistance remains an important clinical challenge. Several different mechanisms of IM have been described. ${ }^{32,37}$

Table I Management of imatinib mesylate (IM) toxicity ${ }^{16,32,33}$

\begin{tabular}{|c|c|}
\hline Interaction with surgery & $\begin{array}{l}\text { - Imatinib (IM) can be stopped right before surgery and restarted as soon } \\
\text { as po medication is tolerated }\end{array}$ \\
\hline Hepatotoxicity & $\begin{array}{l}\text { - Monitor LFT initially then monthly } \times 3 \text { then serially } \\
\text { - Stop if bilirubin } \geq 3 \times \text { IULN or transaminases } \geq 5 \times \text { IULN }\end{array}$ \\
\hline Bone marrow toxicity & $\begin{array}{l}\text { - CBC should be performed weekly for the first month, bi-weekly for the second } \\
\text { month and every } 2-3 \text { months thereafter }\end{array}$ \\
\hline Fluid retention & $\begin{array}{l}\text { - Superficial retention can be managed with diuretics, other supportive measure or } \\
\text { dose reduction }\end{array}$ \\
\hline $\begin{array}{l}\text { Thyroid function } \\
\text { impairment }\end{array}$ & $\begin{array}{l}\text { - Monitor TSH closely and treat as appropriate } \\
\text { - A 2-fold increase in levothyroxine is recommended before initiating treatment }\end{array}$ \\
\hline Drug interactions & $\begin{array}{l}\text { - Caution should be taken if patient is taking inhibitor or inducer of de CYP3A4 } \\
\text { - If large dose of imatinib is prescribed, patient should take no more than I.3 g a day } \\
\text { of acetaminophen (imatinib inhibits ACTM glucurodination) }\end{array}$ \\
\hline
\end{tabular}

Abbreviations: LFT, liver function test; IULN, institutional upper limit of normal; CBC, complete blood count; TSH, thyroid-stimulating hormone; ACTM, acetaminophen. 
Some patients presenting with secondary IM resistance will respond to a dose escalation to $400 \mathrm{mg}$ twice a day. If this strategy fails, SM can be started. SM is a multi-tyrosine kinase inhibitor that has been shown to be active in IMresistant or IM-intolerant patients in a phase III trial. ${ }^{19}$

Patients who progress under IM and SM treatments should be included in ongoing clinical trials. Other tyrosine kinase inhibitors are now being tested for efficacy in GIST treatment. Nilotinib (Tasigna ${ }^{\circledR}$, Novartis) has been shown to be well tolerated and to have clinical activity in IM-resistant GIST. ${ }^{38}$ There are also preclinical studies suggesting an effect of the tyrosine kinase inhibitor sorafenib (Nexavar ${ }^{\circledR}$, Bayer Leverkusen, Germany) on GIST. ${ }^{39,40}$

\section{Combining surgery and IM}

Surgery remains the primary treatment for resectable GIST and offers the only chance of cure. However, IM has an important role to play in patients with increased risk of recurrence. Integration of IM with the treatment paradigm in the adjuvant setting has significantly decreased the recurrence of GIST as demonstrated by ACOSOG Z9001, the phase III trial. However, the impact of this approach on long-term survival remains unclear. In general, IM treatment can be initiated as soon as the patients recover from their surgical procedures, especially in patients with significant risk of recurrence (tumor size $\geq 10 \mathrm{~cm}, \geq 5$ mitoses per $50 \mathrm{HPF}$, rupture, intraperitoneal hemorrhage, and multifocal GIST). The recommended adjuvant therapy is a daily dose of $400 \mathrm{mg}$ for 1 year. The benefits of longer duration therapy remain to be proven. Neoadjuvant IM should not be routinely used, but it should be considered if a downsizing of the tumor would allow a less morbid operation. This should be conducted in a multidisciplinary setting.

For patients presenting with metastatic or recurrent GIST, the exact place of surgery in the IM era remains to be determined. Surgery is generally not indicated if a patient presents with generalized disease progression under treatment. In such situation, the outcomes appear to be marginal: one study reported no patients free of recurrence at 12 months ${ }^{41}$ and another reported a median time to progression of 3 months. ${ }^{42}$ In a patient with diffuse progression, medical therapy is more appropriate. IM dose can be increased up to $400 \mathrm{mg}$ twice a day, and if the disease is resistant to IM, SM can be used, which has been shown to be effective in the phase III trial. ${ }^{19}$

Patients with preoperative response to therapy or at least stable disease seem to do better with 2-year progression-free survival of $60 \%-70 \%{ }^{42,43}$ However, the exact impact of surgery on DFS and OS in this setting is unknown. The prognosis for patients undergoing surgery for limited progressive disease (progression of only one or few metastases with globally responding disease) appears to be slightly better than for patients with generalized progressive disease. ${ }^{41,42}$

\section{Conclusion}

In the treatment of GIST, surgery and IM therapy are two powerful methods that allow us to offer a better prognosis to our patients. For primary resectable GIST, the first step should be the complete excision of the tumor. Neoadjuvant IM therapy can be considered for initially unresectable tumor or if a downsizing of the tumor would allow resection with decreased surgical morbidity. Adjuvant IM should be given to patients at higher risk of recurrence. For patients with metastatic or recurrent GIST, the treatment is based on IM therapy and for resistant or intolerant patients, SM can be used.

Some points remain to be clarified. Notably, the ideal duration of adjuvant IM after surgery is still unclear. It is difficult to determine the exact place of surgery in metastatic or recurrent GIST patients. It is also unclear if surgery makes a difference in outcomes. A multidisciplinary approach and a close collaboration between the medical oncologist, the gastroenterologist, the radiologist, and the surgeon are, therefore, mandatory to offer GIST patients the best treatment available.

\section{Disclosures}

The authors report no conflicts of interest in this work.

\section{References}

1. Hirota S, Isozaki K, Moriyama Y, et al. Gain-of-function mutations of c-kit in human gastrointestinal stromal tumors. Science. 1998;279 (5350): 577-580.

2. Joensuu H, Roberts P, Sarlomo-Rikala M, et al. Effect of the tyrosine kinase inhibitor STI571 in a patient with a metastatic gastrointestinal stromal tumor. N Engl J Med. 2001;344(14):1052-1056.

3. Perez E, Livingstone A, Franceschi D, et al. Current incidence and outcomes of gastrointestinal mesenchymal tumors including gastrointestinal stromal tumors. J Am Coll Surg. 2004;202(4):623-629.

4. Gold JS, DeMatteo R. Combined surgical and molecular therapy: the gastrointestinal stromal tumor model. Ann Surg. 2006;244(2):176-184.

5. Miettinen M, Lasota J. Gastrointestinal stromal tumors: review on morphology, molecular pathology, prognosis, and differential diagnosis. Arch Pathol Lab Med. 2006;130(10):1466-1478.

6. Nikfarjam M, Kimchi E, Shereef S, et al. Surgical outcomes of patients with gastrointestinal stromal tumors in the era of targeted drug therapy. J Gastrointest Surg. 2008;12(11):2023-2031.

7. DeMatteo R, Lewis J, Leung D, Mudan S, Woodruff J, Brennan M. Two hundred gastrointestinal stromal tumors: recurrence patterns and prognostic factors for survival. Ann Surg. 2000;231(1):51-58.

8. Heinrich M, Corless C, Duensing A, et al. PDGFRA activating mutations in gastrointestinal stromal tumors. Science. 2003;299(5607):708-710.

9. Rutkowski P, Nowecki Z, Michej W, et al. Risk criteria and prognostic factors for predicting recurrences after resection of primary gastrointestinal stromal tumor. Ann Surg Oncol. 2007;14(7):2018-2027.

10. Nilsson B, Bümming P, Meis-Kindblom J, et al. Gastrointestinal stromal tumors: the incidence, prevalence, clinical course, and prognostication in the preimatinib mesylate era - a population-based study in western Sweden. Cancer. 2005;103(4):821-829. 
11. Heinrich $\mathrm{M}$, Corless $\mathrm{C}$, Demetri $\mathrm{G}$, et al. Kinase mutations and imatinib response in patients with metastatic gastrointestinal stromal tumor. J Clin Oncol. 2003;21(23):4342-4349.

12. Debiec-Rychter M, Sciot R, Le Cesne A, et al. KIT mutations and dose selection for imatinib in patients with advanced gastrointestinal stromal tumours. Eur J Cancer. 2006;42(8):1093-1103.

13. Heinrich M, Owzar K, Corless C, et al. Correlation of kinase genotype and clinical outcome in the North American Intergroup Phase III Trial of imatinib mesylate for treatment of advanced gastrointestinal stromal tumor: CALGB 150105 Study by Cancer and Leukemia Group B and Southwest Oncology Group. J Clin Oncol. 2008;26(33):5360-5367.

14. Pierie J, Choudry U, Muzikansky A, Yeap B, Souba W, Ott M. The effect of surgery and grade on outcome of gastrointestinal stromal tumors. Arch Surg. 2001;136(4):383-389.

15. Aparicio T, Boige V, Sabourin J, et al. Prognostic factors after surgery of primary resectable gastrointestinal stromal tumours. Eur J Surg Oncol. 2004;30(10):1098-1103.

16. Demetri G, Antonia S, Benjamin R, et al. NCCN Clinical Practice Guidelines in Oncology, Soft Tissue Sarcoma; 2009.

17. Verweij J, Casali P, Zalcberg J, et al. Progression-free survival in gastrointestinal stromal tumours with high-dose imatinib: randomised trial. Lancet. 2004;364(9440):1127-1134.

18. Blanke C, Rankin C, Demetri G, et al. Phase III randomized, intergroup trial assessing imatinib mesylate at two dose levels in patients with unresectable or metastatic gastrointestinal stromal tumors expressing the kit receptor tyrosine kinase: S0033. J Clin Oncol. 2008;26(4):626-632.

19. Demetri G, van Oosterom A, Garrett C, et al. Efficacy and safety of sunitinib in patients with advanced gastrointestinal stromal tumour after failure of imatinib: a randomised controlled trial. Lancet. 2006;368(9544):1329-1338.

20. Gold J, van der Zwan S, Gönen M, et al. Outcome of metastatic GIST in the era before tyrosine kinase inhibitors. Ann Surg Oncol. 2007;14 (1): 134-142.

21. Demetri G, von Mehren M, Blanke C, et al. Efficacy and safety of imatinib mesylate in advanced gastrointestinal stromal tumors. $N$ Engl J Med. 2002;347(7):472-480.

22. Verweij J, van Oosterom A, Blay J, et al. Imatinib mesylate (STI-571 Glivec, Gleevec) is an active agent for gastrointestinal stromal tumours, but does not yield responses in other soft-tissue sarcomas that are unselected for a molecular target. Results from an EORTC Soft Tissue and Bone Sarcoma Group phase II study. Eur J Cancer. 2003; 39(14):2006-2011.

23. Blanke C, Demetri G, von Mehren M, et al. Long-term results from a randomized phase II trial of standard- versus higher-dose imatinib mesylate for patients with unresectable or metastatic gastrointestinal stromal tumors expressing KIT. J Clin Oncol. 2008;26(4):620-625.

24. Zalcberg J, Verweij J, Casali P, et al. Outcome of patients with advanced gastro-intestinal stromal tumours crossing over to a daily imatinib dose of 800 $\mathrm{mg}$ after progression on $400 \mathrm{mg}$. Eur J Cancer. 2005;41(12): 1751-1757.

25. DeMatteo RP, Antonescu CR, Chadaram V, et al. Adjuvant imatinib mesylate in patients with primary high risk gastrointestinal stromal tumor (GIST) following complete resection: safety results from the U.S. intergroup phase II trial ACOSOG Z9000. [abstract]. J Clin Oncol. 2005;23(Suppl 16):A-9009, 818s.

26. Dematteo R, Ballman K, Antonescu C, et al. Adjuvant imatinib mesylate after resection of localised, primary gastrointestinal stromal tumour: a randomised, double-blind, placebo-controlled trial. Lancet. 2009; 373(9669):1097-1104.
27. Eisenberg B, Harris J, Blanke C, et al. Phase II trial of neoadjuvant/ adjuvant imatinib mesylate (IM) for advanced primary and metastatic/ recurrent operable gastrointestinal stromal tumor (GIST): early results of RTOG 0132/ACRIN 6665. J Surg Oncol. 2009;99(1):42-47.

28. Fiore M, Palassini E, Fumagalli E, et al. Preoperative imatinib mesylate for unresectable or locally advanced primary gastrointestinal stromal tumors (GIST). Eur J Surg Oncol. 2009;35(7):739-745.

29. Choi H, Charnsangavej C, de Castro Faria S, et al. CT evaluation of the response of gastrointestinal stromal tumors after imatinib mesylate treatment: a quantitative analysis correlated with FDG PET findings. AJR Am J Roentgenol. 2004;183(6):1619-1628.

30. Choi H, Charnsangavej C, Faria S, et al. Correlation of computed tomography and positron emission tomography in patients with metastatic gastrointestinal stromal tumor treated at a single institution with imatinib mesylate: proposal of new computed tomography response criteria. J Clin Oncol. 2007;25(13):1753-1759.

31. Rubin B, Fletcher J, Fletcher C. Molecular insights into the histogenesis and pathogenesis of gastrointestinal stromal tumors. Int J Surg Pathol. 2000;8(1):5-10.

32. de Kogel C, Schellens JHM. Imatinib. Oncologist. 2007;12(12): 1390-1394.

33. FDA. Gleevec (imatinib mesylate) Revised Product Label 2006.

34. van Oosterom A, Judson I, Verweij J, et al. Safety and efficacy of imatinib (STI571) in metastatic gastrointestinal stromal tumours: a phase I study. Lancet. 2001;358(9291):1421-1423.

35. Tonyali O, Coskun U, Yildiz R, et al. Imatinib mesylate-induced acute liver failure in a patient with gastrointestinal stromal tumors. Med Oncol. 2009 Aug 7. Epub ahead of print.

36. Van Glabbeke M, Verweij J, Casali P, et al. Predicting toxicities for patients with advanced gastrointestinal stromal tumours treated with imatinib: a study of the European Organisation for Research and Treatment of Cancer, the Italian Sarcoma Group, and the Australasian Gastro-Intestinal Trials Group (EORTC-ISG-AGITG). Eur J Cancer. 2006;42(14):2277-2285.

37. Jiang Y, Ming L, Montero A, Kimchi E, Nikfarjam M, StaveleyO'Carroll K. Optimizing imatinib mesylate treatment in gastrointestinal stromal tumors. Gastrointest Cancer Res. 2008;2(5):245-250.

38. Demetri G, Casali P, Blay J, et al. A phase I study of single-agent nilotinib or in combination with imatinib in patients with imatinib-resistant gastrointestinal stromal tumors. Clin Cancer Res. 2009;15(18):5910-5916.

39. Huynh $\mathrm{H}$, Lee J, Chow $\mathrm{P}$, et al. Sorafenib induces growth suppression in mouse models of gastrointestinal stromal tumor. Mol Cancer Ther. 2009;8(1):152-159.

40. Guo T, Agaram N, Wong G, et al. Sorafenib inhibits the imatinib-resistant KITT670I gatekeeper mutation in gastrointestinal stromal tumor. Clin Cancer Res. 2007;13(16):4874-4881.

41. Raut C, Posner M, Desai J, et al. Surgical management of advanced gastrointestinal stromal tumors after treatment with targeted systemic therapy using kinase inhibitors. $J$ Clin Oncol. 2006;24(15):2325-2331.

42. DeMatteo R, Maki R, Singer S, Gonen M, Brennan M, Antonescu CR. Results of tyrosine kinase inhibitor therapy followed by surgical resection for metastatic gastrointestinal stromal tumor. Ann Surg. 2007; 245(3):347-352.

43. Gronchi A, Fiore M, Miselli F, et al. Surgery of residual disease following molecular-targeted therapy with imatinib mesylate in advanced/ metastatic GIST. Ann Surg. 2007;245(3):341-346.
Therapeutics and Clinical Risk Management

\section{Publish your work in this journal}

Therapeutics and Clinical Risk Management is an international, peerreviewed journal of clinical therapeutics and risk management, focusing on concise rapid reporting of clinical studies in all therapeutic areas, outcomes, safety, and programs for the effective, safe, and sustained use of medicines. This journal is indexed on PubMed Central, CAS,

\section{Dovepress}

EMBase, Scopus and the Elsevier Bibliographic databases. The manuscript management system is completely online and includes a very quick and fair peer-review system, which is all easy to use. Visit http://www.dovepress.com/testimonials.php to read real quotes from published authors. 